\title{
Das Mass aller Dinge
}

\author{
Erhard Taverna
}

«Auf Geheiss der Wirtschaft und ihrer hündischen Vollstrecker sei LQS, das lohnwirksame Qualifikationssystem, auch in die Schulstuben eingedrungen.» So räsoniert Loos im neuen Roman «Am Hang» von Markus Werner. «Zwecks Qualifizierung der Lehrperson schneie nämlich von Zeit zu Zeit ein Visitator herein, setze sich hinten in eine Bank, breite diverse Blätter und eine Checkliste vor sich aus und achte während des Unterrichts auf die Fachkompetenz und die Methodenkompetenz und die Sozialkompetenz der Lehrperson, wobei ihm zur kompetenten Beurteilung dieser drei Kompetenzen nicht weniger als neununddreissig Beurteilungskriterien zur Verfügung stünden.» Dazu gehören Gestik und Mimik, Vorbildwirkung, Durchhaltevermögen und Humor. Im erzählten Albtraum bekommt Loos einen Abzug wegen seiner ausgeprägten Nasolabialfalte.

Kummerfalten werden auch im Bulletin der «Vereinigung Schweizerischer Hochschuldozenten» sichtbar [1]. Das Editorial beginnt mit einem Zitat, das vom erwähnten Loos stammen könnte: «Die Ökonomisierung aller Lebensbereiche macht alle nur ärmer.» Thema des Heftes ist die Akkreditierung im Hochschulbereich und die mangelnde Vertretung der Dozenten in den zahlreichen Instanzen der Forschungs- und Bildungspolitik. Das jüngste Seilziehen zwischen Bund und Kantonen um die Finanzierung der Universitäten hat das komplizierte Räderwerk dieser Instanzen für die Dauer einiger Schlagzeilen blossgelegt.

Das Thema ist nicht nur für die Evaluation von Studiengängen aktuell. Gemäss Beschluss der europäischen Bildungsminister sollen bis 2005 in allen Ländern ein System der Akkreditierung, der Zertifizierung oder ähnliche Verfahren eingerichtet werden. Eine Praxis, die für Spitäler, auf freiwilliger Basis, schon begonnen hat. Worum geht es dabei?

Es geht um ein formales und transparentes Verfahren, das anhand definierter, international kompatibler Standards Qualitätsanforderungen überprüft. Das obligatorische Gütesiegel der dafür geschaffenen Agenturen entscheidet über die Zulassung von Lehrangeboten, die Anerkennung von Diplomen und Titeln und die Finanzierung. Ziel sei es, den Bildungsmarkt zu regulieren und die Mobilität der Studierenden für die neuen Bachelor- und Masterstudiengänge $\mathrm{zu}$ fördern.
Auch Spitäler und Praxisbetriebe werden aufgrund dieser Standortanalyse Stärken und Schwächen entdecken und ihr Angebot anpassen. Quervergleiche sind ein Gewinn, wenn sie nötige Verbesserungen und Reformen zur Folge haben. Was im Hochschulbereich als Chancen und Grenzen, als Mehrwert oder Bürokratie diskutiert wird, betrifft genau gleich das Gesundheitswesen in der Schweiz. Auch hier gilt für die Zertifizierung der dreistufige Ablauf von Selbstbeurteilung, Vor-Ort-Visite durch externe Experten und Akkreditierungsentscheid. Zur Diskussion stehen die Kriterien eines durchstrukturierten Verfahrens, das auf wirtschaftlichen Wettbewerb und Konkurrenz ausgerichtet ist. Ob Auftrag und Ertrag der aufwendigen und kostspieligen Evaluation, die zudem periodisch zu wiederholen ist, in einem angemessenen Verhältnis stehen, ist umstritten.

Das Neue daran ist eine Steuerungspraxis mit dem Anspruch, die Leistung von Bildungsund Gesundheitssystemen messbar zu machen. Dafür soll es inhaltsneutrale und technisch perfekte Verfahren geben, die alle wirtschaftlichen und politischen Konsequenzen legitimieren. Die Aufgabenerfüllung, gemessen an formalisierten Prozessen, gewichtet Organisation und Management höher als die Professionalität der im System tätigen Fachpersonen. Kritisiert wird die alleinige Legitimation durch ein Verfahren, das nur zählt, was zugänglich ist. Zeitknappheit, Zwang zur Auswahl an Informationen und Gesprächspartnern, die eigene limitierte Aufnahmefähigkeit und vor allem die Notwendigkeit, am Ende etwas auszusagen, führten zu Willkür und Ungerechtigkeit. Das Verfahren sei der Sache gegenüber indifferent, man könne es zwischen Fremden verwenden, ohne sich um das Besondere zu kümmern. Die Macht des überwachenden Expertentums erzwinge neben der bisherigen, auf das eigene Wissen bezogenen Fachsprache eine zusätzliche Programmsprache, was den früheren Inszenierungsaufwand an Legitimation weit übertreffe. Befürchtet wird, dass im Repertoire des Zählens und Quantifizierens menschliche und wissenschaftliche Werte verlorengehen. Bedauert wird auch die Begünstigung eines Kollegentyps, der seine fachliche Anerkennung und seine Karriere weniger der herkömmlichen Professionalität als der geschickten Anpassung an die neuen Machtverhältnisse verdankt. Das wäre allerdings, mit oder ohne Nasolabialfalte, nichts Neues. 